\title{
INCORPORANDO LOS RIESGOS DEL SIGLO XXI EN LA VALUACIÓN DE MARCAS
}

\author{
LUIS FERNANDO SAMPER ${ }^{*-* *}$
}

\section{INTRODUCCIÓN}

Hace unas décadas se desarrolló un consenso sobre la importancia de los bienes intangibles en la creación de valor ${ }^{1}$. La "curva de la sonrisa" (figura 1) ilustra que las principales actividades generadoras de valor se sitúan en las actividades de concepción y desarrollo de los productos y en las de distribución y mercadeo, ámbitos asociados con las patentes, los secretos empresariales y los signos distintivos, respectivamente, en tanto que las actividades estrictamente asociadas con la producción no son las mayores generadoras de valor. En consecuencia, miles de empresas mantienen un control férreo sobre sus intangibles intensivos en conocimiento y actividades de mayor valor agregado, como investigación y desarrollo o mercadeo, mientras que contratan o subcontratan actividades de producción estandarizables con maquiladoras o con proveedores con acceso a mercados laborales de costos inferiores.

\footnotetext{
* Economista y máster en Derecho de la Universidad de los Andes y máster en Administración de Negocios de Columbia University - NY. Actualmente es director de 4.0 Brands. Bogotá, D. C., Colombia. Correo electrónico: Luis.samper@4point0brands.com. Para citar el artículo: SAMper, L. F. "Incorporando los riesgos del siglo XXi en la valuación de marcas", Revista La Propiedad Inmaterial n. ${ }^{\circ}$ 24, Universidad Externado de Colombia, julio-diciembre 2017, pp. 27-51. DoI: https://doi.org/10.18601/16571959.n24.02

** Agradezco a los doctores Luis Carlos Pombo, Ernesto Rengifo y José Luis Londoño por sus comentarios a versiones preliminares de este documento. Así mismo, agradezco a Juan Fernando Giraldo y al equipo de Búho Media por sus contribuciones. María Alejandra Echavarría, de la Pontificia Universidad Bolivariana, y Carlos A. Conde, de la Universidad Externado de Colombia, también tuvieron la generosidad de leer y comentar el manuscrito. Por supuesto, cualquier error u omisión de esta versión es de mi exclusiva responsabilidad.

1 Ver, entre otros, Mudambi, Ram. Location, control and innovation in knowledgeintensive industries. Journal of Economic Geography. Oxford: Oxford University Press, 2008, 8(5), pp. 699-725. issn: 1468-2702; Rengifo, Ernesto y Ромbo, Luis Carlos. Valuación de activos intangibles de propiedad intelectual. Bogotá: Universidad Externado de Colombia, 2015. isbn: 9789587724165; Torres, Leonidas. La importancia de los activos intangibles en la sociedad del conocimiento. Revista La Propiedad Inmaterial. Bogotá: Universidad Externado de Colombia, 2004, (18), pp. 5-34. IssN: 1657-1959.
} 
Figura 1. La curva de la sonrisa

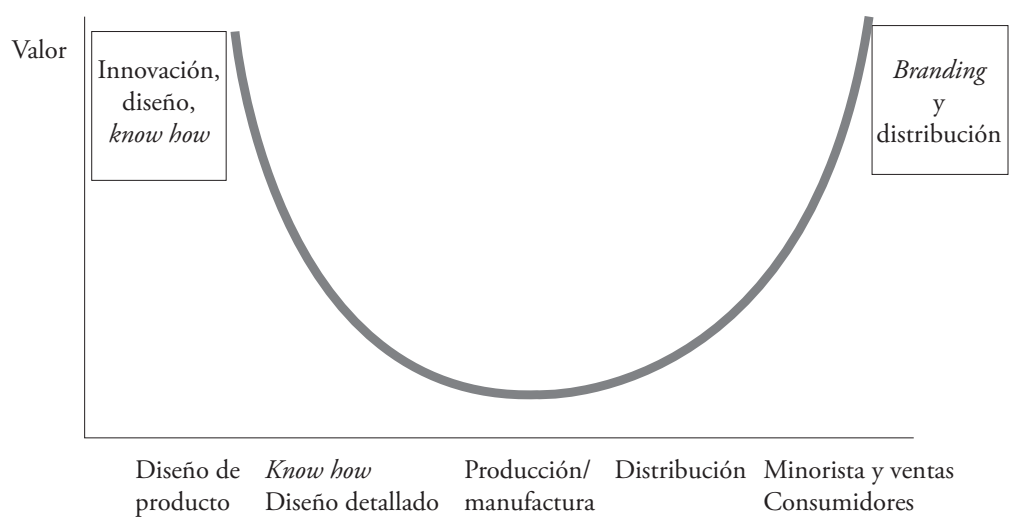

Fuente: Fallows, James. China makes, the world takes. The Atlantic Magazine [en línea], julio/agosto, 2007

De esta forma, con la globalización del comercio de mercancías se generaron cadenas de valor para diferentes industrias y prestadores de servicios en múltiples países. México, Taiwán, India o China -una vez este país protocolizó su adhesión a la Organización Mundial del Comercio-son los ejemplos más frecuentes de esta dinámica. Aun en aquellos casos en los que surgen nuevos competidores globales provenientes de países en desarrollo ${ }^{2}$, la literatura sugiere que para estos nuevos jugadores tanto la innovación como el mercadeo son cruciales en la dinámica de la apropiación de valor.

La globalización y el comercio internacional de mercancías trajeron consigo nuevos retos no solo en materia de coordinación de cadenas de valor, proveedores y ensamblaje, sino también en la necesidad de generar confianza. Productos originarios de diferentes geografías requirieron el acompañamiento a proveedores confiables no solo en términos de calidad, sino también en términos de responsabilidad empresarial. Diversas noticias sobre abusos en materia laboral o ambiental, de riesgos de inocuidad o de trabajadores esclavos, entre muchos otros riesgos, pueden afectar drásticamente los negocios de marcas y organizaciones. El reciente escándalo de la carne débil proveniente de Brasil ${ }^{3}$ es el último de una larga historia de eventos a los que se enfrentan las compañías globales que distribuyen productos provenientes de diferentes geografías, eventos que han generado un mayor escepticismo por parte de consumidores y grupos de interés frente a las grandes marcas y que han favorecido a los actores pequeños que pueden demostrar más

2 Ver PanAnond, Pavida. Breakout multinationals: Emerging-market multinationals in global value chains. En M. Demirbag \& A. Yaprak (eds.). Handbook of emerging market multinational corporations. Northampton, MA: Edward Elgar Publishing, 2015. ISBN: 978-1782544999.

3 Ver ввс. Lo que se sabe del escándalo en Brasil con la carne podrida que era "maquillada" para ser exportada, 2017. Disponible en http://www.bbc.com/mundo/noticiasamerica-latina-39327633 
fácilmente su cercanía con la comunidad ${ }^{4}$. A raíz de estos eventos, es claro que las nuevas generaciones y la sociedad en general se han tornado más exigentes frente a las compañías y marcas, sobre sus estándares de responsabilidad social y ambiental, requiriendo mayor transparencia y políticas de sostenibilidad más elaboradas.

El acceso a la información y la forma como la sociedad se expresa también tienen serias implicaciones en el devenir de los negocios de las marcas. Contrario a lo que sucedía hace un par de décadas, en el siglo xxi las marcas no controlan la información que se genera sobre ellas. Las redes sociales, los medios digitales, los blogs o las campañas de ONG pueden afectar sensiblemente la percepción sobre una industria, el valor de una marca y los patrones de demanda sobre sus productos. Basta recordar la reciente evolución de la industria de gaseosas, mejor conocida hoy como la industria de las bebidas azucaradas, o la aparente sanción social para quienes aún utilizan pitillos ${ }^{5}$ para consumir bebidas. En un mundo donde las percepciones pueden generar complejas realidades ${ }^{6}$, muchas de las narrativas en redes sociales y medios digitales pueden convertirse en tendencias que deban enfrentar las marcas, tendencias que crean cambios drásticos en los patrones de demanda e influyen incluso en las regulaciones. Este conjunto de narrativas o micronarrativas entre diversos actores de la sociedad, que en este documento se denominan "el diálogo social", son identificables en los espacios digitales y los medios tradicionales y claramente son una fuente potencial de riesgo para las marcas que debe ser tenida en cuenta. Así, el diálogo social se constituye en un elemento fundamental que ha de ser tomado en consideración por las empresas y las marcas que quieran ser relevantes en el largo plazo desde la perspectiva tanto de sus plataformas de comunicación como del tipo de productos o servicios que ofrecen.

Por otra parte, también es cierto que las organizaciones empresariales que están mejor preparadas e incluso pueden participar activamente en este diálogo social son aquellas que se han tomado el trabajo de desarrollar e implementar políticas y reportes sofisticados de sostenibilidad. Cuando se tiene una visión clara de las vulnerabilidades y de los retos en materia de sostenibilidad, se puede desarrollar un contenido claro y presentarlo de forma transparente a una sociedad que cada vez espera más de las empresas, reduciendo de esta manera los riesgos y vulnerabilidades que enfrentan sus marcas.

Ante este panorama conviene hacer un alto en el camino para evaluar cómo los cambios que ha traído consigo el fenómeno de la globalización, las mayores exigencias de los consumidores y la sociedad, las tendencias de la tecnología, las

4 Ver Samper, Luis F.; Giovanucci, Daniele y Marques Vieira, Luciana. The powerful role of intangibles in coffee value chains: Emerging solutions to grow value and distribute it more equitably [wIPo Economic Research Working Paper n. ${ }^{3}$ 39], noviembre de 2017.

5 El término pitillo se utiliza en Colombia y Venezuela para las pajillas, popotes o pajita en otros países hispanos.

6 Ver Bryant, Jennings \& Zillman, Dolf. Media effects: Advances in theory and research. Mahwah, NJ: LEA Publishers, 2002. ISBN: 9780805838633, en particular, SHRUM, LARRY J. "Media consumption and perceptions of social reality - Effects of Underlying processes” en dicha publicación. 
comunicaciones y la responsabilidad social han tenido un impacto en el valor de los intangibles y deben ser considerados en las metodologías que se utilizan para su valuación. En este trabajo se sostiene que los modelos de valuación de marcas tradicionales no incorporan los riesgos característicos de este nuevo entorno, y se proponen las adaptaciones necesarias para llevar a cabo valuaciones que reflejen más acertadamente los retos y riesgos actuales que enfrentan las marcas. Se trata de actualizar las metodologías de valuación diseñadas en el siglo xx para las nuevas realidades del siglo XXI, donde el "diálogo social" puede ser un determinante sobre la capacidad de las empresas y sus marcas para generar valor consistentemente en el tiempo, y puede además afectar las regulaciones que las rigen. El argumento es que se debe ser más específico a la hora de aplicar un valor adicional asociado con el riesgo de marca o el Beta de marca (o Beta adicional de industria, para aquellas que son vulnerables al diálogo social).

Este artículo está dividido en siete secciones, comenzando por la presente introducción. En la siguiente sección se hace un breve recuento sobre algunos de los nuevos retos y mayores riesgos que, en opinión del autor, enfrentan las marcas en el entorno de los negocios en el siglo xxi. A continuación, se ilustra cómo los métodos de valuación actualmente utilizados no incorporan los riesgos asociados con este nuevo contexto. En la cuarta sección se ilustra una aproximación metodológica para cuantificar estos nuevos retos y sus atenuantes. Posteriormente, se introduce el concepto de resiliencia de marca y su posible uso para incorporar en las metodologías de valuación, así como las posibles formas de cuantificar los riesgos o la vulnerabilidad legal de una marca. Finalmente, se concluye con algunas anotaciones sobre la importancia de actualizar los modelos existentes hoy a las nuevas realidades.

\section{UN MUNDO GLOBALIZADO E INTERDEPENDIENTE}

\section{DONDE EL DIÁLOGO SOCIAL PUEDE AFECTAR LA CAPACIDAD DE OPERAR}

La globalización y la forma como los individuos se expresan y se informan han generado nuevos riesgos para las marcas y que no se han tenido en cuenta de una manera formal en las metodologías tradicionales de valuación de intangibles. Las fuentes de dichos riesgos son básicamente tres: una cadena de valor y de suministro larga, cuyos estándares son difíciles de controlar y verificar ${ }^{7}$; cambios significativos en el rol de las empresas en la sociedad ${ }^{8}$; $y$ una mayor vigilancia y empoderamiento

7 Ver, entre otros, Gereffi, Gary. Global production systems and Third World development. In B. Stallings (ed.). Global change, regional response: The new international context of development. Cambridge: Cambridge University Press, 1995. IsBn: $978-$ 0521478069 y Fallows, James. China makes, the world takes. The Atlantic Magazine [en línea], julio/agosto, 2007.

8 Ver Zadek, Simon. The path to corporate responsibility. Harvard Business Review [en línea]. Boston, MA: Harvard Business School Publishing, 2004, 82. Issn: 0017-8012. Disponible en https://hbr.org/2004/12/the-path-to-corporate-responsibility_(accesado el 2 enero de 2017) y Williams, Freya. Green giants. How smart companies turn sustainability 
de los clientes y consumidores por medio de espacios digitales ${ }^{9}$, que pueden generar cambios en la demanda y en las regulaciones.

En lo que tiene que ver con las cadenas de valor, es claro que muchas empresas han diversificado sustancialmente sus fuentes de aprovisionamiento ${ }^{10}$. Desde la perspectiva financiera, las empresas y los encargados de las cadenas de suministro y distribución que han adaptado sus cadenas de aprovisionamiento a los requerimientos de la globalización han logrado reducir el capital de trabajo comprometido en la producción de bienes, optimizando sus inventarios de materias primas y producto terminado y mejorando sus indicadores de gestión de caja. Este fenómeno comenzó a consolidarse a partir de la década de los noventa, cuando los sistemas de manejo de inventarios "justo a tiempo" tomaron más fuerza, pero a la vez implicó mayor dependencia con los proveedores.

Esta mayor dependencia conlleva riesgos reputacionales para las empresas en un mundo hiperconectado, que se caracteriza principalmente por un consumidor empoderado y un empleado exigente ${ }^{11}$. En la realidad del siglo xxi predomina un inquisitivo escepticismo que exige transparencia en cuanto al origen y la forma de transformar los productos ${ }^{12}$, y las nuevas generaciones (de empleados y consumidores) han creado nuevos retos para las empresas y las marcas desde la perspectiva de la información que deben proveer para mantener su licencia social para operar. Las fallas en las cadenas de suministro (desde la perspectiva económica, social, ambiental) se convierten rápidamente en noticias difundidas por los medios o por los mismos consumidores o empleados, que pueden fácilmente afectar la reputación de una marca ${ }^{13}$.

into billion-dollar business. New York: Amacom, 2015. ISBN: 978-0814436134.

9 Roberts, Kevin. Lovemarks the future beyond brands. New York: Powerhouse Books, 2005. isBn: 978-1576872703 y Mainwaring, Simon. We first: How brands and consumers use Social Media to build a better world. New York: St Martin's Press, 2011. ISBN: 0230110266 .

10 Ver, entre otros, Contractor, Farok; Kumar, Vikas; Kundu, Sumit K. \& PederSEn, Torben. Reconceptualizing the firm in a world of outsourcing and offshoring: The organizational and geographical relocation of high-value company functions. Journal of Management Studies, 2010, 47(8), pp.1417-1433. IsSN: 1467-6486. Ver también BRAT, Ilan \& Gasparro, Annie. El origen de los ingredientes se vuelve una prioridad para las empresas de alimentos. Wall Street Journal [en línea], 17 de marzo de 2016. ISSN: 0099-9660. Disponible en https://www.wsj.com/articles/el-origen-de-los-ingredientes-sevuelve-una-prioridad-para-las-firmas-de-alimentos-1458258439

11 Ver Nielsen. The sustainability imperative. New insights on consumer expectations. New York, NY: The Nielsen Company. 2015. Disponible en http://www.nielsen.com/ content/dam/nielsenglobal/dk/docs/global-sustainability-report-oct-2015.pdf y DELOITTE. The 2016 Deloitte Millennial Survey: Winning over the next generation of leaders. Disponible en https://www2.deloitte.com/global/en/pages/about-deloitte/articles/millennialsurvey.html

12 LABEL Insight. How consumer demand for transparency is shaping the food industry. The 2016 Label Insight Food Revolution Study. Chicago IL: Label Insight, 2016. Disponible en https://www.labelinsight.com/food-revolution-study?__hssc=11 $7566870.1 .1505083065568 \&$ \&_hstc $=117566870.9911 \mathrm{~d} 2 \mathrm{~d} 67 \mathrm{bd} 62 \mathrm{f0ec} 4 \mathrm{cfcaaf0} 1 \mathrm{da} 1 \mathrm{f}$ df. $1505083065568.1505083065568 .1505083065568 .1 \&$ \&_hsfp $=2491176415 \& \mathrm{hsCt}$ aTracking $=80 \mathrm{c} 3102 \mathrm{a}-\mathrm{e} 5 \mathrm{e} 8-4 \mathrm{cc5}-960 \mathrm{~b}-\mathrm{afb} 14 \mathrm{dc5b} 6 \mathrm{c0} \% 7 \mathrm{C} 8211592 \mathrm{e}-92 \mathrm{~b} 4-4349-9653-$ 55345 bdc6288 y Brat y Gasparro, op. cit.

13 Di Somma, Mark. Will customers forgive your brand? 18 de abril de 2016. Disponible 
Es claro que este nuevo contexto genera mayores riesgos para las marcas y, en consecuencia, afecta su valuación e incluso el valor mismo de las empresas. En esta era es imposible aislarse de las fallas o de los escándalos que puedan surgir en materia social o ambiental de un proveedor, puesto que la sociedad espera que las empresas y sus marcas hagan cumplir unos estándares mínimos a toda su cadena de valor, independientemente de la cadena de valor que se utilice. Para atenuar dichos riesgos, las marcas buscan mantener su credibilidad y paralelamente tener un mayor conocimiento sobre sus complejas cadenas de suministro, lo que implica acciones proactivas para asegurar que sus proveedores cumplan los estándares legales, sociales y éticos. Por otra parte, las organizaciones deben estar innovando constantemente para cumplir los mayores requisitos ambientales y ecológicos que los consumidores, en particular los jóvenes o millennials, les exigen ${ }^{14}$.

Desde la perspectiva del rol de las empresas en la sociedad, también es necesario tener en cuenta que las empresas ya no son solo concebidas como proveedores de bienes o servicios, sino como actores sociales. Claramente, las formas de relacionarse entre la empresa y la comunidad han cambiado de manera sustancial desde 1970, cuando Milton Friedman aseguraba que la responsabilidad social de los negocios era simplemente incrementar sus utilidades ${ }^{15}$. Luego de décadas de experiencias en iniciativas de responsabilidad social corporativa (CSR) y gobernanza, ambiente y sociedad (ESG), actualmente se espera que las marcas cumplan un propósito que vaya mucho más allá de ser rentables, agregando valor a la sociedad y a las comunidades que impactan ${ }^{16}$. Así, en la medida en que en el siglo xxi el sector privado asume un rol protagónico en la implementación de estrategias sociales y ambientales como parte de su rol empresarial, los riesgos reputacionales de las marcas no se limitan a posibles fallas en la cadena de suministro. En otras palabras, es evidente que las expectativas de la sociedad frente a la contribución de las marcas y empresas al bienestar social han aumentado.

Una forma de realizar diagnósticos y focalizar prioridades para atender las demandas sociales y reducir los riesgos es por medio de la realización de informes de sostenibilidad. Es ya común encontrar entre empresas exitosas la publicación de sus prioridades en materia de sostenibilidad en temas económicos, ambientales y sociales, que en el pasado se consideraron del ámbito exclusivo de los gobiernos ${ }^{17}$.

en http://www.brandingstrategyinsider.com/2016/04/will-customers-forgive-your-brand. html\#.VxfCQ_nhdiu; Edmans, Alex; Lucius, Li \& Zhang, Chendi. Employee satisfaction, labor market flexibility, and stock returns around the world [Finance Working Paper n. ${ }^{\circ}$ 433/2014]. Brussels: European Corporate Governance Institute (ECGI), 2014.

14 Nielsen. Op. cit. Mainwaring, Simon. Op. cit.

15 Friedman, Milton. The social responsibility of business is to increase its profits. The New York Times Magazine [en línea], 13 de septiembre de 1970. Issn: 0028-7822.

16 Williams, Freya. Op. cit.

17 Ver, entre otros, KPMG International. Sustainable insight. The essentials of materiality assessment, 2016. Disponible en https://www.kpmg.com/Global/en/IssuesAndInsights/ ArticlesPublications/Documents/materiality-assessment.pdf; Khan, Mozaffar; Serafeim, George \& Yoon, Aaron. Corporate sustainability: First evidence on materiality [Working Paper 15-073]. Boston: Harvard Business School, 2015 y Gidwani, Bahar. The link be- 
En este entorno, las grandes empresas y los inversionistas exigen informes de sostenibilidad más sofisticados, con metodologías elaboradas, como las del Global Reporting Initiative (GRI) o el Sustainability Accounting Standards Board (SASB). De hecho, algunas compañías han comenzado a reportar su contribución al logro de los Objetivos de Desarrollo Sostenible (oDs) de las Naciones Unidas en sus informes de sostenibilidad, lo que claramente ilustra cómo han cambiado sustancialmente las expectativas de la sociedad frente al rol de las empresas.

Una tercera fuente de vulnerabilidad tiene que ver con el empoderamiento de los consumidores y los grupos de interés gracias a la tecnología. En la medida en que diferentes actores pueden acceder y compartir información que puede afectar la reputación de las marcas, sus patrones de demanda e incluso la actitud de los reguladores hacia las industrias de donde provienen pueden cambiar. En el mundo del siglo XXI, las marcas enfrentan mayores requisitos de transparencia y complejos retos de comunicación con clientes y consumidores que pueden ser influenciados por informaciones de diferentes fuentes y con variados niveles de credibilidad. Los tiempos que describen los textos clásicos de mercadeo y publicidad, cuando la marca controlaba sus comunicaciones y se dirigía a sus clientes y consumidores por medio de publicidad tradicional, son cosa del pasado.

En el entorno actual, las marcas son apenas un actor en la comunicación que se genera sobre ellas mismas y sus industrias ${ }^{18}$. Estos riesgos se han incrementado sustancialmente en la era de las redes sociales, en la medida en que una proporción significativa de consumidores utiliza este medio como fuente primaria de información. Los teóricos de la "cognición social" sostienen que las personas fundan sus juicios con base en la información a la que pueden acceder fácilmente, y que con base en dichos juicios se realizan acciones por parte de los consumidores, tomadores de decisión o reguladores ${ }^{19}$. Este principio de la cognición social está relacionado con la accesibilidad, es decir, qué tipo de información para tomar decisiones es relativamente accesible, lo que ratifica la importancia de los medios y las redes sociales como fuentes de información para llevar a cabo juicios que puedan alterar tendencias y comportamientos. Además del acceso a la información, un segundo elemento para realizar juicios tiene que ver con la cantidad de información que los individuos consideran necesaria para realizar sus juicios. En un mundo hiperconectado, donde la gente no tiene capacidad para procesar o entender a cabalidad temas complejos y mucho menos juzgar la calidad de la información que recibe para formarse una opinión sobre una marca o empresa, las personas toman decisiones con un volumen de información limitado. Este proceso, conocido como el

tween brand value and sustainability. The Conference Board, Oct 2013. Disponible en http:// brandfinance.com/images/upload/the_link_between_brand_value_and_sustainability.pdf

18 Ver Di Somma, Mark. Op. cit. y Roberts. Op. cit.

19 Ver Shrum, Larry J. Op. cit. 
principio heurístico o de suficiencia, supone que las fuentes en muchos casos se limitan a los conocidos, los medios digitales y las redes sociales ${ }^{20}$.

En otras palabras, los consumidores y la sociedad en general se han vuelto cada vez más influenciados e influenciables por lo que sucede en los medios digitales y en las redes sociales para tomar posiciones y juicios sobre marcas, empresas o industrias. Basta recordar las consultas ciudadanas sobre las actividades mineras para entender la dimensión que estos fenómenos pueden tener en las estrategias de las empresas.

El diálogo social del siglo XXI es, en consecuencia, una fuente adicional de riesgo sobre las industrias, las empresas y sus marcas, que debe ser considerado cuidadosamente. En la medida en que es precisamente por medio del diálogo social que se presentan las narrativas que conducen a formar juicios que determinan cambios en los comportamientos, y en que las marcas no controlan el diálogo social que las afecta, han aumentado considerablemente los riesgos de que las percepciones (reales o manipuladas) que se construyen en los ámbitos digitales sobre una marca o una industria puedan generar cambios en las tendencias, así como nuevas y complejas realidades de negocio. Las marcas en el siglo Xxi deben ser conscientes de que los consumidores, mediante su diálogo social, intercambian información, aumentan sus exigencias y requieren acción preventiva.

A manera de ejemplo, casos como la marca Odebrecht contribuyeron a consolidar una narrativa alrededor del tema de la corrupción en el sector de infraestructura que rápidamente ha contagiado a otros sectores y ha puesto la corrupción como un tema central del diálogo social, llevando a las autoridades y a los reguladores a buscar demostrar acciones al respecto. Así, el valor de las marcas asociadas con infraestructura se vio alterado por un contagio "intrasectorial" que condujo a que los cierres financieros para otras compañías en diferentes proyectos se complicasen. Paralelamente, se produjo un contagio "intersectorial" asociado con percepciones o realidades relacionadas con el tema de corrupción, motivadas por el diálogo social sobre este tema, que ha afectado a marcas y empresas de otras industrias, incrementando sus riesgos de operación y su forma de hacer negocios. Similares tipos de contagio asociado con retos reputacionales de otras industrias, como la de bebidas azucaradas, se han presentado para toda la industria de alimentos, que ahora debe prestar especial atención a los ingredientes y a los atributos nutricionales de sus productos. Es claro entonces que el diálogo social puede tener consecuencias significativas para diferentes marcas e industrias.

Es así como los cambios en el entorno han alterado las fuentes de los riesgos de las marcas. Una sociedad vigilante y empoderada, que se expresa por medio del diálogo social, implica mayores riesgos que es necesario incorporar en las valuaciones de intangibles y de las empresas en general. Es evidente que un inversionista, el

20 En este sentido, las personas no utilizan toda la información disponible para realizar sus juicios, lo que conduce implícitamente a juicios sesgados por el tipo de medios que utilizan para informarse. 
sector financiero o un potencial franquiciatario o licenciatario de una marca van a requerir no solo entender estas vulnerabilidades, sino estar en capacidad para cuantificarlas. Ahora bien, ¿`cómo se deben medir dichos riesgos? ¿Cuáles son sus impactos en los ejercicios de valuación? Este tema se desarrollará en la siguiente sección.

\section{MIDIENDO LOS RIESGOS PARA PROPÓSITOS DE VALUACIÓN}

Los modelos tradicionales de valoración asociados con el llamado "enfoque ingreso" incluyen un indicador de riesgo vinculado a la tasa de descuento utilizada para calcular, en precios de hoy, los flujos relacionados con el ingreso esperado del negocio o de la marca. Es así como una mayor tasa de descuento -es decir, un mayor riesgo- implica un menor valor del activo en proceso de valuación.

$\mathrm{Al}$ calcular la tasa de descuento que se va a aplicar a los flujos de ingreso esperados para empresas o industrias específicas, usualmente se utiliza la tasa promedio ponderada del costo de capital utilizado por la empresa o promedio de la industria (Weighted Average Cost of Capital o WACC), tasa que refleja el costo ponderado de la deuda y del mercado de accionistas relevante para la empresa. Esta tasa es utilizada regularmente por banqueros de inversión y por los administradores para evaluar la rentabilidad de sus proyectos. Un componente clave del WaCc es el Capital Asset Pricing Model (CAPM), que refleja el retorno esperado del accionista y que utiliza, con base en las cotizaciones de las acciones de las compañías comparables o de la industria en cuestión, la prima de riesgo que exige un accionista para invertir en la compañía o industria analizada.

A la prima de riesgo de la industria a la que pertenece la empresa o la marca en el CAPM se le conoce como el factor Beta ( $\beta$ de industria), indicador que busca reflejar el riesgo de que dicha industria o compañía no genere el flujo de ingresos predecibles que un accionista en esa industria requiere. Por ejemplo, la inversión de un accionista en una compañía grande en el sector de alimentos es percibida como menos riesgosa que una acción de una compañía de productos de lujo, en la medida en que la demanda por alimentos va a ser menos elástica a cambios en el ingreso de los consumidores resultante de cambios en el ciclo económico, en tanto que la demanda por productos de lujo seguramente se verá afectada en mayor medida por una recesión. Se infiere en este ejemplo que las acciones en el sector de alimentos son menos volátiles y más seguras, con un Beta de industria de alimentos menor, aunque quizá no necesariamente sean las más rentables en el largo plazo.

Cuando se valoran activos intangibles como una marca, la tasa de descuento utilizada es regularmente superior al WACC, es decir, incorpora riesgos adicionales. Esta mayor tasa se explica por los mayores riesgos del activo valorado. Teniendo en cuenta que el retorno esperado de un intangible es mayor que el de un activo fijo reemplazable, la tasa de descuento que realmente refleja la rentabilidad esperada de una marca no fácilmente reemplazable debe ser superior al wACC de la empresa en 
cuestión. Así, tanto la rentabilidad esperada como el indicador de riesgo implícito en la tasa de descuento que se va a utilizar deben ser superiores al WACC y reflejar así no solo los riesgos implícitos capturados por el Beta de la industria, sino los riesgos del intangible, en este caso, la marca que se va a valorar.

En otras palabras, en las tasas de descuento utilizadas para una gran parte de los modelos de valoración de intangibles ${ }^{21}$ se utiliza un Beta de industria que refleja principalmente los riesgos sistémicos de la economía en el sector analizado, pero no se incluyen los riesgos adicionales asociados con los intangibles. Conscientes de esta problemática, algunos autores adicionan cifras entre 1 y $4 \%$ al WACC asociado con ciertos activos intangibles por valorar que se consideran inherentemente más riesgosos ${ }^{22}$, en tanto que otros han utilizado una cifra genérica de $2 \%$ que adicionan a la tasa de descuento WACC promedio de la compañía para reflejar riesgos específicos adicionales de una marca. A este valor se le conoce como Beta de marca o valor adicional al WACC, que refleja los riesgos asociados con dicho intangible.

El Beta de marca en el siglo xxi está íntimamente relacionado con la percepción y los juicios que hagan los grupos de interés de la marca o su industria sobre su devenir y la confianza que ella misma genere. Es claro que, en el contexto actual, las narrativas asociadas con una marca pueden afectar la reputación de sus productos e incluso la demanda de toda una industria. Así, para propósitos de este artículo, el término diálogo social se utiliza en un sentido más amplio que el de otros autores que lo han tratado desde la perspectiva laboral o de los derechos de autor ${ }^{23}$. Se trata de identificar patrones generales de diálogo que permitan entender la forma como la sociedad percibe o habla sobre las marcas y las industrias, y a la vez cuantificar la capacidad de influencia sobre los juicios que se hacen sobre estas. Es por medio de este diálogo como la sociedad define tanto la reputación como la relevancia de las marcas y las industrias en el futuro.

Al defender la idea de que el Beta de marca en el siglo xxi está íntimamente ligado a las narrativas asociadas con la empresa o la industria en cuestión, se hace necesario medir el impacto que dicho diálogo puede tener en el devenir de los negocios de la marca, para poder ser más específicos a la hora de aplicar un valor

21 Salinas y Perrier describen diferentes metodologías utilizadas en valuación de marcas. Estas descripciones están fuera del ámbito de este artículo. Basta decir que en la gran mayoría de los casos se calcula el valor presente de la marca mediante el uso de una tasa de descuento que refleja el riesgo implícito en el activo valorado, en este caso, la marca. Perrier, Raymon \& Stobart, Paul (eds.). Brand valuation. Londres: Premier Books, 1997. ISBN: 978-1900617000. SAlinas, Gabriela. Valoración de marcas. Barcelona: Ediciones Deusto, 2007. ISBN: 9788423425211. Ромвo, Luis Carlos. Estándares de valuación de activos intangibles de propiedad intelectual. Bogotá: Universidad Externado de Colombia, 2015. ISBN: 9789587723458.

22 Mard, Michael; Hitchner, James \& Hyden, Steven. Valuation for financial reporting. 2. ${ }^{a}$ ed. Hoboken NJ: John Wiley and Sons, 2007. ISBN: 978-0-471-68041-3.

23 Elkin-Koren, Niva. Copyright law and social dialogue on the information superhighway: The case against copyright liability of bulletin board operators. Cardozo Arts \& Entertainment Law Journal. New York: Benjamin N. Cardozo School of Law, 1994, 13. Disponible en http://heinonline.org/HoL/LandingPage?handle=hein.journals/ caelj $13 \&$ div $=32 \&$ id $=\&$ page $=$ 
adicional asociado con el riesgo de marca. En consecuencia, los valuadores deben justificar el Beta de marca, que en la actualidad es un genérico de alrededor del $2 \%$, y su monto debe afinarse para que refleje efectivamente una medición de riesgo justificada con datos concretos. En otras palabras, se trata de incorporar con mayor claridad los riesgos asociados con operar en el siglo xxi derivados de la exposición al diálogo social, que sean específicos a cada marca o industria y que claramente varían entre industrias y compañías.

Paralelamente, se ha de considerar que los riesgos del diálogo social pueden ser atenuados en alguna medida por la reputación adquirida y por las políticas de relacionamiento con la sociedad, reflejadas en los informes de sostenibilidad. La reputación está asociada con la capacidad de la marca para recuperarse frente a crisis inesperadas o escándalos que la afecten, es decir, con su capacidad de resiliencia. Este tema será explorado en la quinta sección de este artículo. En lo que tiene que ver con la forma de enfrentar cotidianamente los riesgos asociados con el diálogo social, la seriedad de las políticas de sostenibilidad es una forma de generar contenido relevante para participar proactivamente en los diálogos alrededor de la marca y la industria. Así, la calidad de los informes de sostenibilidad se constituye en un instrumento estratégico para dar las respuestas creíbles que exigen los grupos de interés de las marcas, y en especial los empleados y consumidores modernos. Por esta razón, una política elaborada de sostenibilidad no solo tiene un efecto positivo en la reputación organizacional, sino que también puede influir positivamente en la valoración de los intangibles de la compañía e incluso en la compañía en su conjunto.

\section{I Formalizando EL MOdelo}

Se pasa ahora a ilustrar el argumento de una forma esquemática. Para cuantificar los riesgos de diálogo social, vale la pena retomar los componentes del WACC y del CAPM que calculan la tasa de descuento que refleja los riesgos específicos de la industria. El WACC es el promedio ponderado de las dos fuentes de financiación de la empresa: los accionistas y la deuda (neta de impuestos). El costo de capital de los accionistas está determinado por el CAMP y se expresa de la siguiente forma:

(2) CAMP $\quad \mathrm{r}=\mathrm{rf}+\mathrm{CR}+[(\mathrm{Rm}-\mathrm{rf}) \times(\beta$ industria $)]$

donde

rf: tasa de interés libre de riesgo.

$\mathrm{Rm}$ : tasa de interés de mercado aplicable a la compañía o industria.

CR: country risk (o spread). 
$\beta$ Industria: refleja el riesgo sistémico de cambios en el ciclo económico en la industria en cuestión.

Sin embargo, de acuerdo con la exposición hecha en el presente artículo, la tasa de descuento que se va a utilizar para propósitos de valoración de marcas debe incorporar otros elementos que reflejen los mayores riesgos de diálogo social mencionados o que los atenúen. De esta manera, se diferencia el riesgo sistémico de la industria ( $\beta$ industria) frente al riesgo de marca específico asociado con el diálogo social relacionado con la industria o marca analizada o $\beta$ marca. En consecuencia, el componente del CAMP corregido incorporaría al menos los siguientes elementos:

(3) CAMP corregido $=r f+C R+[(R m-r f) \times(\beta$ industria $)]+(\beta$ marca $)$

donde,

$\beta$ marca $=$ riesgo de marca . Refleja el riesgo asociado con la marca relacionado con el diálogo social sobre la marca o sobre la industria a la que pertenece y sus posibles atenuantes en materia de sostenibilidad.

Esta fórmula tiene dos partes: la tasa de descuento aplicable para la empresa o industria derivada del camp y el riesgo asociado con el riesgo de marca, y se puede simplificar como se indica en la siguiente sección, en la que se hace un recuento de la propuesta del autor sobre metodologías de cálculo para dichos riesgos.

\section{CALCULANDO EL RIESGO DE MARCA}

Como se ha mencionado, el riesgo de marca tiene al menos dos componentes: el riesgo de diálogo social (RDS) sobre la marca o industria y el índice de fortaleza en sostenibilidad (IFS). El RSD incluye temas actuales o prospectivos que la marca o industria debe enfrentar como consecuencia de las percepciones o cambios que pueda generar dicho diálogo en los patrones de demanda. Por su parte, el IFs es un atenuante del RDs y busca medir la capacidad de respuesta de la marca o compañía específica a temas que la puedan afectar en lo que está ocurriendo en el RDs.

(3) $\beta$ marca $=f($ RDS, IFS $)$

Se describe a continuación la forma como se calculan estos riesgos.

\section{I Riesgo DE DiÁlogo SOCIAL}

RDS es el riesgo de diálogo social, que incorpora un índice de riesgo derivado del impacto de las conversaciones en medios digitales y redes sociales sobre la marca o industria específica que puedan conllevar riesgos en los patrones de demanda o 
cambios en las condiciones regulatorias. Esta variable asume que las percepciones tienen el riesgo de volverse realidades que impactan la dinámica de la marca y sus ventas, y busca identificar las presiones futuras que la marca o industria deberá enfrentar para seguir satisfaciendo las necesidades y los requisitos de los clientes o consumidores.

En primer lugar, es importante subrayar que el riesgo de que las percepciones se conviertan en realidades no es menor. Teóricos de la economía han sugerido que las personas ejercen una racionalidad limitada o acotada para tomar decisiones, y en ese sentido no son totalmente racionales para tomar decisiones, ya sea por falta de información, falta de tiempo u otro tipo de limitaciones ${ }^{24}$. Como se señaló antes, en el campo de las comunicaciones los teóricos de la cognición social también argumentan que los individuos y la sociedad en su conjunto realizan juicios con base en información incompleta -muchas veces imperfecta o incluso falsa- a la que fácilmente tienen acceso.

En este sentido, no solo es necesario evaluar la información que circula en el diálogo social y que pueda afectar a las empresas, sino su capacidad de generar una gran difusión que pueda afectar realmente las estrategias de negocio o la demanda por una marca particular, discerniendo entre eventos particulares y tendencias de mediano y largo plazo. En suma, consolidar unas métricas de riesgo de diálogo social para las marcas y empresas es, sin duda, un reto singular al cual se le han dedicado meses de trabajo y sobre el que se están reportando avances significativos en este artículo.

\section{El modelo Foresight}

Para calcular el RDS, se han analizado las posibles fuentes de información relevante y diferentes aproximaciones metodológicas. De los miles de menciones y titulares en medios digitales y redes sociales, es evidente que los más poderosos y que tienen mayor difusión, consistentes con el principio de accesibilidad de la cognición social, son aquellos en los que se presentan simultáneamente actores que originan o determinan una acción o acusación - positiva o negativa, comprobable o no comprobable- y una víctima o destinatario en esa misma observación o contenido. De esta manera, el universo de análisis más significativo está compuesto por micronarrativas presentes en los contenidos, puesto que tienen una mayor capacidad de contagio y de formar procesos de cognición social. Estas micronarrativas llevan, en todo caso, un generador de riesgo (activo, según nuestro análisis), que puede ser el ente, la persona, el vocero, la marca o la industria que genera un diálogo que involucra un riesgo o una oportunidad sobre una compañía, industria, persona o sociedad. A este receptor del riesgo se le denomina pasivo. 
La unidad de análisis asociada con micronarrativas permite partir de diálogos que tienen algún nivel de "contagio" y hace posible medir los riesgos involucrados en el diálogo social con una metodología clara. De esta manera se acota el volumen de información disponible para identificar, entre los centenares de miles de titulares y artículos de prensa y de conversaciones de redes sociales, cuáles son los temas que pueden constituirse en una tendencia, riesgo o amenaza para una marca o una industria. En ese sentido, es necesario aplicar principios de Smart Data para analizar y cualificar la información, aplicando los tamices y filtros necesarios para poder identificar dichas tendencias o riesgos empresariales que realmente puedan afectar la capacidad de generar valor del intangible.

Estos tamices o filtros deben responder a temas clave para las marcas o las empresas o industrias que se van a analizar, como mercadeo engañoso, privacidad, corrupción o nutrición, entre muchos otros. Una vez conocidos los temas centrales, se pueden identificar los riesgos para la marca que tengan el potencial de desarrollar percepciones negativas sobre el producto o la industria y que puedan constituirse, con el tiempo, en un riesgo latente, emergente, consolidado o institucionalizado para la marca o la industria en cuestión, lo que implica niveles de respuesta y preparación diferentes para las empresas ${ }^{25}$. Ahora bien, no todas las micronarrativas o los fragmentos de contenido analizados necesariamente conducen a riesgos. El cálculo del índice de RDs debe también tener en cuenta que algunas de ellas pueden ser neutrales tanto para actores como para víctimas, e incluso pueden conllevar oportunidades para estas últimas.

La capacidad para que las diferentes micronarrativas alrededor de un tema específico puedan constituirse en un riesgo u oportunidad relevante está también asociada con su potencial de difusión. Para determinar su capacidad de contagio de los diferentes contenidos, se analiza su potencial para generar emoción, para aumentar la credibilidad o "moneda social" a quien lo comparte ${ }^{26}$, el valor práctico o la usabilidad de compartirlo y la popularidad o actualidad sobre el contenido compartido $^{27}$. Al aplicar cinco de los seis principios de contagio de $\mathrm{Berger}^{28}$, se tiene una muy relevante evaluación de las posibilidades de "infección" de posibles riesgos de diálogo social intra e interindustria de una narrativa.

Se han tenido avances significativos en la cuantificación del RDs. En un ejercicio realizado entre enero y septiembre de 2017 se analizaron las narrativas asociadas con cinco temas clave: corrupción, mercadeo engañoso, nutrición, privacidad y seguridad en los productos en cerca de 3 millones de artículos en medios digitales

25 Sadek, Simon. Op. cit.

26 Es decir, el efecto que proyecta sobre quien comparte la información, el hecho de compartirla.

27 Berger, Jonah. Contagious - Why things catch on. New York, NY: Simon \& Schuster, 2013. ISBN: 978-1451686586.

28 Por dificultad práctica no se incluye en el análisis el principio de activación (triggers) de Berger en la evaluación de la posibilidad de contagio de una pieza de contenido específica. 
colombianos. Aplicando los parámetros descritos anteriormente, esta metodología -desarrollada por Búho Media y 4.0 Brands, y a la que se denomina Foresight (previsión) - permite medir los riesgos que enfrentan las marcas y empresas asociados con el diálogo social que se presenta en medios digitales y redes sociales.

$\mathrm{Al}$ aplicar simultáneamente los principios de cognición social, las narrativas y su capacidad de contagio y la categorización de riesgos, se está avanzando significativamente en entender y cuantificar los fenómenos sociales que pueden afectar a las marcas o a las industrias. El siguiente reto es cuantificar esos riesgos para introducirlos en los análisis de valuación de marcas. La metodología Foresight hace posible medir el nivel de riesgo sobre una marca o industria a partir de un índice construido en el que se involucran tres variables de análisis que, en la suma del conjunto, brindan una medida de letalidad de cada historia que la afecta.

La primera de estas variables tiene que ver con el ya explicado nivel de contagio, el cual explica cómo los productos, las ideas y los comportamientos se pueden difundir como "epidemias sociales" en una población. La segunda de estas variables retrata el tipo de viralidad tangible en redes sociales ${ }^{29}$, a la que se le dio el nombre de viralidad manifiesta. Este es un tipo de viralidad que describe el comportamiento de una historia en redes sociales, desde el momento en el que nace y hasta el momento en el que pierde relevancia. Y como última variable está la denominada variable de victimización, la cual responde a la necesidad del proyecto de darle un valor diferencial a las historias que cuentan con unos generadores y receptores de riesgo visibles que impactan en una mayor medida en las licencias sociales de las empresas.

De esta manera, Foresight permite medir efectivamente los riesgos involucrados en el diálogo social que consolidan las percepciones de la sociedad y pueden convertirse en realidades, y de esa manera tener una aproximación más específica al llamado Beta de marca. Es además concebido entonces como un proceso de innovación que permite un análisis de contenido por medio del cual se detectan señales y patrones indicativos de las crisis, de tal forma que facilita su anticipación y control. Por esta razón, también se utiliza como instrumento de medición que puede darle un giro a la prevención de riesgos o a la preparación de alguna medida defensiva para anticiparse y minimizar un daño que es posible que ocurra en empresas, marcas e industrias.

\section{3 ÍNDiCe De ForTALeZa De SOSTENibilidad (IFS)}

El IFs es el índice de fortaleza de sostenibilidad, que mide la capacidad para poder proveer información relevante y estructurada asociada con la sostenibilidad económica, social y ambiental de las operaciones de la marca. Diversos estudios han buscado demostrar la correspondencia entre una sólida política de sostenibilidad 
y el valor de mercado de una empresa ${ }^{30}$, y se ha encontrado una clara correlación entre políticas de sostenibilidad avanzadas y valor de empresa. De hecho, las marcas más valiosas de Colombia tienen reportes de responsabilidad social y sostenibilidad relativamente completos ${ }^{31}$, que incluyen los aspectos ambientales, sociales y económicos de la operación.

Sin embargo, valorar los informes de sostenibilidad más allá del mero cumplimiento de los requisitos técnicos que exigen las diversas metodologías es una labor que no se hace regularmente. La propuesta que se expone en este artículo busca valorar los informes en tres dimensiones: transparencia, compromiso e impacto. Por transparencia se entiende la profundidad del análisis de materialidad del reporte de sostenibilidad. En este sentido, la empresa o la marca debe hacer un juicioso análisis de sus principales retos frente a los de sus pares de la industria o frente a industrias similares, consultando internamente y a sus grupos de interés sobre sus desafíos inmediatos y de largo plazo ${ }^{32}$. El compromiso se refiere a la relevancia de las metas establecidas frente al tamaño de la empresa y sus prioridades, y el impacto tiene que ver con la evaluación de su gestión respecto a los propósitos planteados. Se detallan a continuación cada una de las variables mencionadas.

En el marco de los análisis de sostenibilidad de las empresas, un elemento clave está asociado con el llamado análisis de materialidad en lo relativo a sostenibilidad. La definición de las prioridades estratégicas en materia de sostenibilidad con la suficiente profundidad para poder desarrollar las acciones pertinentes con los impactos esperados es un elemento clave que está asociado con la calificación de transparencia. Sin un análisis robusto de materialidad, va a ser difícil mantener la licencia ambiental y social para operar y, además, conectar efectivamente con sus clientes y empleados alrededor de un propósito compartido.

Un completo análisis de materialidad debe conllevar una definición de políticas y objetivos en esta materia que la empresa y la marca deben emprender, con metas cuantificables. El compromiso busca medir si dichas metas son acordes con el tamaño del negocio y el impacto que los diferentes temas tienen en él. Por

30 Ver, entre otros, Morgan, STANLEY. Sustainable reality. Understanding the performance of sustainable investment strategies. New York: Morgan Stanley Institute for Sustainable Investing, 2015. Disponible en http://www.sustainablebrands.com/digital_learning/research_report/ stakeholder_trends_insights/sustainable_reality_understanding_perfo; Sustainable Brands. 22 Research studies proving the ROI of sustainability. San Francisco, CA: Sustainable Brands, 2016. Disponible en http://e.sustainablebrands.com/resources-report-22-research-studiesproving-the-roi-of-sustainability.html?_ga=1.77425757.974955321.1467725336\&iesrc= ctr; WBCsD (World Business Council for Sustainable Development) \& UNEP FI (United Nations Environment Programme Finance Initiative). Translating ESG into sustainable business value: Key insights for companies and investors. Report from an International Workshop Series of the WBCSD and Unep FI, unep FI: Geneva, Switzerland. ConchesGeneva, Switzerland: UNP and WBCSD, 2010. Disponible en http://www.unepfi.org/ fileadmin/documents/translatingESG.pdf; Williams, Freya. Op. cit.

31 Ver Samper, Luis F. Marcas y sostenibilidad, ¿hacia dónde vamos? Portafolio [en línea], 27 de septiembre de 2016. Issn: 0123-6326. Disponible en http://www.portafolio. co/negocios/especial-marcas-en-colombia-2016-500622+

32 La metodología de Foresight anteriormente descrita puede ser otra fuente de información y detección de asuntos materiales de sostenibilidad. 
ejemplo, una política de donaciones que no sea relevante con la sostenibilidad del negocio o aportes o gestos simbólicos ${ }^{33}$ que no tienen una relación clara con los retos enunciados no tendrán una calificación necesariamente positiva. Así, la calidad de las acciones, los objetivos y las metas en materia de sostenibilidad es crucial para evaluar la calidad del reporte.

La tercera variable que se debe considerar es el impacto de las acciones desarrolladas. En este sentido, tener claras metodologías de medición de impacto demuestra el interés de perfeccionar sus políticas y su trabajo en lo relativo a la sostenibilidad. De esta manera, al calificar estas tres categorías favorablemente se atenúan los riesgos que en materia de diálogo social enfrentan las marcas, pues estarán en condiciones de sostener conversaciones profundas y convincentes con sus públicos de interés en diferentes materias y temas.

\section{VULNERABILIDAD O RIESGO LEGAL}

Un componente adicional que se debe considerar en la cuantificación del valor de una marca está relacionado con la fortaleza de los registros legales y su distintividad en el mercado (mercados), que puedan limitar sus posibilidades de desarrollo o expansión. Este riesgo puede limitar sus capacidades de desarrollo en diferentes mercados o segmentos o en diferentes industrias, y puede hacer a la marca más vulnerable a las estrategias de su competencia.

Es entonces evidente que una mayor vulnerabilidad legal o una menor distintividad pueden afectar el valor del intangible, así como una patente puede ser más o menos valiosa, dependiendo de cuán bien redactadas estén sus reivindicaciones. En este sentido, es claro que el rol del especialista en propiedad intelectual es clave y agrega valor a las empresas. El reto, sin embargo, es cuantificar ese valor.

Desde la perspectiva de la cuantificación, la vulnerabilidad legal o fortaleza de la protección podría ser considerada como un tercer componente del riesgo de marca, elevando la tasa de descuento WACC, o podría alternativamente afectar el valor del intangible reduciendo la rentabilidad esperada o la capacidad de crecimiento del negocio por la vía del cálculo de los ingresos esperados bajo una metodología de valuación basada en el "enfoque ingreso".

La vulnerabilidad legal en primer lugar va a depender, en función de la jurisdicción o territorio, del volumen esperado de operaciones y de la rentabilidad de la marca en cada territorio, de las controversias existentes, de la seguridad jurídica del sistema de propiedad industrial (en términos de observancia y de predictibilidad

33 Un ejemplo de un gesto simbólico puede ser una compañía que reforeste una hectárea de tierra cuando es un consumidor de agua industrial masivo que implicaría un activo programa de mantenimiento de cuencas y microcuencas en su zona de influencia. El anuncio de la reforestación de la hectárea, aunque consistente con sus asuntos materiales relacionados con el uso del agua en materia ambiental, es claramente un esfuerzo de relaciones públicas que no tiene correlación con la dimensión de sus retos ambientales. 
de las decisiones de la autoridad respectiva) y de los posibles acuerdos de coexistencia o de su ausencia en la marca que se va a analizar. Así mismo, declaraciones de notoriedad $\mathrm{u}$ otras formas que fortalezcan los derechos legales van a reducir esta vulnerabilidad, sin duda.

En lo que se refiere a la distintividad inherente o adquirida de la marca, esta debe cuantificar el riesgo de que esta se pierda. En efecto, cuanto más se acerque la marca al producto o a sus características, la empresa tendrá que soportar marcas similares o empresas que evoquen conceptos similares que podrán tener un efecto en las ventas o en los márgenes de operación. En ese sentido, se pueden presentar casos en los cuales la marca en cuestión utilice conceptos, términos o colores que se vuelven tendencia y que por lo mismo queden expuestos a que otros los usen, e inclusive a que se vuelvan un término genérico en el lenguaje del país.

Por otra parte, también puede darse el caso de una limitada distintividad de la marca en diferentes mercados o en la misma industria, de manera que afecte sus posibilidades de expansión. A manera de ejemplo, en Colombia la marca Caracol, utilizada tanto por radio como por televisión en un mismo país y en una misma industria, puede no ser lo suficientemente distintiva para quienes la operan y limitar sus posibilidades de crecimiento en diferentes segmentos e industrias. Lo mismo puede suceder con la marca Corona, con presencia significativa en diferentes industrias, pero con diferentes dueños.

En suma, los riesgos o vulnerabilidades legales se pueden evaluar desde la perspectiva de la fortaleza de la protección positiva y de su distintividad. La calificación que reciba cada uno de estos componentes podrá afectar tanto el flujo de ingresos como la rentabilidad de la operación en los mercados de interés, lo cual definitivamente afectará la valuación de este activo intangible. En consecuencia, es claro el rol del asesor legal en reducir estos riesgos y, por ende, en aumentar el valor de la marca.

\section{LA RESILIENCIA DE MARCA}

Otro factor que ha de considerarse en la valuación de marcas es lo que aquí se llama resiliencia de marca. La experiencia ha demostrado que algunas marcas son más resistentes a escándalos y a retos en materia de sostenibilidad. Es evidente que lo sucedido con las marcas Volkswagen en lo que tiene que ver con las "trampas" en la cuantificación de emisiones o con Samsung en lo relacionado con las fallas de seguridad de algunos de sus productos ha afectado en alguna medida el valor de sus negocios y operaciones. Sin embargo, estas marcas han demostrado su capacidad de recuperar sus mercados y rentabilidad con el paso del tiempo, pese a los escándalos en que se han visto envueltas. La "resiliencia" que han demostrado estas marcas contrasta con marcas más pequeñas y menos relevantes que pueden perder más rápidamente la confianza de sus públicos clave y a las cuales les es más difícil recuperarse de situaciones similares. En otras palabras, las marcas tradicionales y consolidadas tienen una mayor resiliencia que las pequeñas y de nicho. Así, se 
denomina resiliencia de marca a la capacidad de sobreponerse a un evento o a una serie de eventos que afecten la confianza en la marca.

En consecuencia, la resiliencia de una marca está relacionada con su tamaño y tradición. Este tipo de marcas tienen además un elemento en común: han incorporado en su quehacer diario filtros y políticas de sostenibilidad que, además de hacerlas más atractivas para inversionistas y más competitivas en el largo plazo ${ }^{34}$, permiten detectar o corregir situaciones que puedan eventualmente generar una crisis reputacional. En adición, los recursos y la capacidad de reacción frente a una situación de escándalo por lo general, aunque no siempre, son superiores en organizaciones de mayor tamaño frente a marcas con participación de mercado menor.

En este sentido, existen dos elementos que pueden afectar la resiliencia. En el corto plazo son fundamentales las acciones que una organización realice frente al evento, y que sus decisiones se perciban como transparentes frente a reguladores y públicos de interés. Desde luego que poder predecir cómo reaccionará una marca o una empresa frente a un escándalo es difícil y probablemente complejo de cuantificar, pero una buena evaluación de su informe de sostenibilidad puede ser un indicador positivo.

Un segundo elemento de resiliencia tiene que ver con la confianza que la marca ha generado a lo largo de los años. Este índice refleja la tradición y fortaleza en el mercado, y de esta manera refleja también la capacidad de una marca de sobreponerse a un escándalo o situación adversa. En este sentido, indicadores como años de operación y participación del mercado constituyen buenos predictores de resiliencia. Por esta razón, más que un riesgo, la resiliencia debe reflejar la capacidad de una marca de generar ingresos por un período prolongado. Así, en la medida en que el indicador de resiliencia es mayor, mayor puede ser el número de períodos de ingresos proyectados derivados de la marca, afectando de esta forma su valuación positivamente.

\section{CONCLUSIONES}

El valor total de las empresas modernas está compuesto, en una gran proporción, por la valoración de sus intangibles. Ahora bien, los intangibles de las empresas, en particular de las marcas, son más vulnerables porque los mercados y la sociedad en general en el siglo xxi son más exigentes y escépticos respecto a las marcas

34 Ver eccles, Robert G.; Ioannou, Ioannis \& Serafeim, George. The impact of corporate sustainability on organizational processes and performance. Management Science. Catonsville, Maryland: Informs, 2014, 60(11), pp. 2835-2857. Issn: 1526-5501 (Online). Disponible en http://www.hbs.edu/faculty/Publication\%20Files/ssRn-id1964011_6791edac7daa-4603-a220-4a0c6c7a3f7a.pdf; Gidwani, BAHAR. Op. cit.; GRI (Global Reporting Initiative). G4 Sustainability Reporting Guidelines, 2016. Disponible en https://www. globalreporting.org/information/g4/Pages/default.aspx; y Khan, Mozaffar; Serafeim, George \& Yoon, Aaron. Corporate sustainability: First evidence on materiality [Working Paper 15-073]. Boston: Harvard Business School, 2015. 
tradicionales, en particular frente a las que tienen cadenas de valor complejas. Esta dinámica de mercado e innovación responde a nuevas realidades de mercado en las que los consumidores, los reguladores y aun los empleados de las organizaciones están en permanente conversación sobre la contribución de las marcas y productos al bienestar común. En un contexto donde la sociedad es más exigente y escéptica en cuanto a la contribución de las marcas a estas nuevas exigencias, los riesgos de mantener la confianza y la credibilidad han aumentado.

$\mathrm{Al}$ construir modelos de valuación modernos se deben tener en cuenta los cambios en el entorno y los mayores riesgos de operación y de mantenimiento de la licencia social y ambiental para operar que requiere la sociedad moderna. En este breve escrito se plantean algunas posibilidades de cómo acometer el reto de valorar marcas en el difícil entorno actual.

Aunque la valuación de intangibles no es una ciencia exacta, así como tampoco lo es la valuación de empresas, es claro que las metodologías tradicionales desarrolladas en el siglo Xx deben adaptarse a las nuevas realidades del siglo XXI. Una consecuencia de esta afirmación es que las tasas de descuento que se utilizan para valuar las marcas en el contexto actual deben reflejar más acertadamente estos nuevos riesgos. Es claro que quienes compren o licencien una marca deben ser conscientes de los posibles debates y retos que podrían enfrentar en el mundo moderno, y que esos riesgos deben reflejarse en el valor del intangible objeto de la negociación, por lo que es indispensable adaptar las metodologías de valuación a las nuevas realidades.

Para acometer este reto, se han introducido algunos nuevos conceptos que, se espera, sean útiles para los valuadores de intangibles y de empresas. En primer lugar, se ha desarrollado una metodología a la que se le ha denominado Foresight, que permite cuantificar los riesgos asociados con el diálogo social sobre las marcas $\mathrm{o}$ las industrias a las que pertenecen y sustanciar los riesgos adicionales que puedan generar tendencias y cambios en los negocios y que deben ser tenidos en cuenta en los procesos de valuación de intangibles. Es claro que en el contexto actual, los medios tradicionales y las redes sociales, donde se expresa un diálogo social que las marcas no controlan, hacen a las marcas vulnerables a rumores o noticias que se contagian y que adquieren una difusión y relevancia inesperadas. Con esta metodología se cuantifican estos riesgos, que se han llamado riesgos de diálogo social específicos a las marcas o a las industrias a las que pertenecen.

También se ha introducido el concepto de la calidad de los informes de sostenibilidad como un elemento clave para demostrar la capacidad de atenuar los riesgos de diálogo social con respuestas serias y elaboradas frente a eventuales cuestionamientos o para ser parte proactiva de ese diálogo.

Adicionalmente, se ha desarrollado una aproximación para cuantificar los riesgos legales en las valuaciones, así como formas de calcular la capacidad de resiliencia que pueda tener una marca frente a eventos que puedan atentar contra la continuidad de su negocio, el acceso a canales de distribución o capacidad de 
recuperar la confianza de reguladores y clientes para poder tener valuaciones de marcas más acertadas.

\section{REFERENCIAS}

Aaker, David. Managing brand equity. New York: Free Press, 1991. IsBN: 9780029001011.

ввС. Lo que se sabe del escándalo en Brasil con la carne podrida que era "maquillada" para ser exportada, 2017. Disponible en http://www.bbc.com/mundo/noticiasamerica-latina-39327633

Berger, Jonah. Contagious - Why things catch on. New York, NY: Simon \& Schuster, 2013. ISBN: 978-1451686586.

Berman, Bruce (ed.). Making innovation pay. NJ: John Wiley and Sons. Hoboken, 2006. ISBN: 978-0471733379.

Brat, Ilan \& Gasparro, Annie. El origen de los ingredientes se vuelve una prioridad para las empresas de alimentos. Wall Street Journal [en línea], 17 de marzo de 2016. IssN: 0099-9660. Disponible en https://www.wsj.com/ articles/el-origen-de-los-ingredientes-se-vuelve-una-prioridad-para-las-firmasde-alimentos-1458258439

Bryant, Jennings \& Zillman, Dolf. Media effects: Advances in theory and research. Mahwah, NJ: LEA Publishers, 2002. ISBN: 9780805838633.

Contractor, Farok; Kumar, Vikas; Kundu, Sumit K. \& Pedersen, Torben. Reconceptualizing the firm in a world of outsourcing and offshoring: The organizational and geographical relocation of high-value company functions. Journal of Management Studies, 2010, 47(8), pp. 1417-1433. ISSN: 1467-6486.

Davis, Scotт. Brand asset management. San Francisco: Jossey Bass, 2002. IsBN: 978-0-7879-6394-1.

Dedrick, JASON \& Kraemer, Kenneth. Intangible assets and value capture in global value chains: The smartphone industry - Mimeo, 2017.

DeloitTe. The 2016 Deloitte Millennial Survey: Winning over the next generation of leaders. Disponible en https://www2.deloitte.com/global/en/pages/aboutdeloitte/articles/millennialsurvey.html

Di Somma, Mark. Will customers forgive your brand? 18 de abril de 2016. Disponible en http://www.brandingstrategyinsider.com/2016/04/will-customersforgive-your-brand.html\#.VxfCQ_nhDIU

Eccles, Robert G.; Ioannou, Ioannis \& Serafeim, George. The impact of corporate sustainability on organizational processes and performance. Management Science. Catonsville, Maryland: Informs, 2014, 60(11), pp. 2835-2857. IssN: 1526-5501 (Online). Disponible en http://www.hbs.edu/faculty/Publication\%20Files/ssRN-id1964011_6791edac-7daa-4603-a220-4a0c6c7a3f7a.pdf 
Edmans, Alex; Lucius, Li \& Zhang, Chendi. (2014). Employee satisfaction, labor market flexibility, and stock returns around the world [Finance Working Paper ${ }^{\circ}{ }^{\circ}$ 433/2014]. Brussels: European Corporate Governance Institute (ECGI), 2014.

Elkin-Koren, Niva. Copyright law and social dialogue on the information superhighway: The case against copyright liability of bulletin board operators. Cardozo Arts \& Entertainment Law Journal. New York: Benjamin N. Cardozo School of Law, 1994, 13. Disponible en http://heinonline.org/Hol/ LandingPage?handle=hein.journals $/$ caelj13\&div=32\&id=\&page $=$

Fallows, James. China makes, the world takes. The Atlantic Magazine [en línea], julio/agosto, 2007.

Friedman, Milton. The social responsibility of business is to increase its profits. The New York Times Magazine [en línea], 13 de septiembre de 1970. IssN: 0028-7822.

GerefFi, Gary. Global production systems and Third World development. In B. Stallings (ed.). Global change, regional response: The new international context of development. Cambridge: Cambridge University Press, 1995. IsBn: 978-0521478069.

Gereffi, Gary; Humphrey, John; Kaplinsky, Raphael \& Sturgeon, Timothy. Introduction: Globalization, value chains and development. IDS Bulletin, 2001, 32(3), pp. 1-8. Online ISsN: 1759-5436.

Gereffi, Gary; Humphrey, John \& Sturgeon, Timothy. The governance of global value chains. Review of International Political Economy. UK: Routledge, 2005, 12(1), pp. 78-104. ISSN: 0969-2290.

GiDWANI, BaHAR. The link between brand value and sustainability. The Conference Board, Oct 2013. Disponible en http://brandfinance.com/images/upload/ the_link_between_brand_value_and_sustainability.pdf

GRI (Global Reporting Initiative). G4 Sustainability Reporting Guidelines, 2016. Disponible en https://www.globalreporting.org/information/g4/Pages/default.aspx

Guerrero, José Manuel. Normas internacionales de información financiera, NIIF. Bogotá: Universidad Externado de Colombia, 2015. IsBN: 9789587724370.

Hubbard, Douglas. How to measure anything. 3. ${ }^{a}$ ed. Hoboken, NJ: John Wiley and Sons, 2014. ISBN: 978-1118539279.

IDC. Data Tracker database on the smartphone industry, 2005-2015. Boston, MA: International Data Corporation, 2016.

Keller, Kevin. Strategic brand management: Building, measuring and managing brand equity. Upper Saddle River, NJ; Prentice Hall, 1998. IsBn: 9780131201156.

Khan, Mozaffar; Serafeim, George \& Yoon, Aaron. Corporate sustainability: First evidence on materiality [Working Paper 15-073]. Boston: Harvard Business School, 2015.

KPMG International. Sustainable insight. The essentials of materiality assessment, 2016. Disponible en https:/www.kpmg.com/Global/en/IssuesAndInsights/ 
ArticlesPublications/Documents/materiality-assessment.pdf. (Accesado el 29 de octubre de 2016).

Label Insight. How consumer demand for transparency is shaping the food industry. The 2016 Label Insight Food Revolution Study. Chicago IL: Label Insight, 2016. Disponible en https://www.labelinsight.com/food-revolution-study?_hssc= $117566870.1 .1505083065568 \&$ __hstc $=117566870.9911 \mathrm{~d} 2 \mathrm{~d} 67 \mathrm{bd} 62 \mathrm{f0ec}$ 4cfcaaf01da1fdf.1505083065568.1505083065568.1505083065568.1\& hsfp $=2491176415 \&$ hsCtaTracking $=80 \mathrm{c} 3102 \mathrm{a}-\mathrm{e} 5 \mathrm{e} 8-4 \mathrm{cc} 5-960 \mathrm{~b}-$ afb14dc5b6c0\%7C8211592e-92b4-4349-9653-55345bdc6288

Landes, William \& Posner, Richard. The economic structure of intellectual property law. Cambridge, MA: Harvard University Press, 2003. IsBN: 9780674012042.

MaInwaring, Simon. We first: How brands and consumers use Social Media to build a better world. New York: St Martin's Press, 2011. ISBN: 0230110266.

Mard, Michael; Hitchner, James \& Hyden, Steven. Valuation for financial reporting. 2. ${ }^{a}$ ed. Hoboken NJ: John Wiley and Sons, 2007. IsBN: 978-0-471-68041-3.

Morgan, Stanley. Sustainable reality. Understanding the performance of sustainable investment strategies. New York: Morgan Stanley Institute for Sustainable Investing, 2015. Disponible en http://www.sustainablebrands.com/digital_learning/ research_report/stakeholder_trends_insights/sustainable_reality_understanding_perfo

MudAMBI, Ram. Location, control and innovation in knowledge-intensive industries. Journal of Economic Geography. Oxford: Oxford University Press. 2008, 8(5), pp. 699-725. IssN: 1468-2702.

Nielsen. The sustainability imperative. New insights on consumer expectations. New York, NY: The Nielsen Company. 2015. Disponible en http://www. nielsen.com/content/dam/nielsenglobal/dk/docs/global-sustainability-reportoct-2015.pdf .

Pananond, Pavida. Breakout multinationals: Emerging-market multinationals in global value chains. En M. Demirbag \& A. Yaprak (eds.). Handbook of emerging market multinational corporations. Northampton, MA: Edward Elgar Publishing, 2015. ISBN: 978-1782544999.

Perrier, Raymon \& Stobart, Paul (ed). Brand valuation. Londres: Premier Books, 1997. ISBN: 978-1900617000.

Poltorak, Alexander \& Lerner, Paul. Essentials of intellectual property. 1. a ed. New York: John Wiley and Sons, 2002. IsBN: 9780471273295.

Pомво, Luis Carlos. Estándares de valuación de activos intangibles de propiedad intelectual. Bogotá: Universidad Externado de Colombia, 2015. ISBN: 9789587723458.

Rengifo, E. Propiedad intelectual: el moderno derecho de autor. 2. ${ }^{a}$ ed. Bogotá: Universidad Externado de Colombia, 1997. IsBN: 9789586162777. 
Rengifo, Ernesto y Роmbo, Luis Carlos. Valuación de activos intangibles de propiedad intelectual. Bogotá: Universidad Externado de Colombia, 2015. ISBN: 9789587724165.

Roberts, Kevin. Lovemarks the future beyond brands. New York: Powerhouse Books, 2005. ISBN: 978-1576872703.

Salinas, Gabriela. Valoración de marcas. Barcelona: Ediciones Deusto, 2007. ISBN: 9788423425211.

SAmper, Luis F. Marcas y sostenibilidad, ¿hacia dónde vamos? Portafolio [en línea], 27 de septiembre de 2016. IssN: 0123-6326. Disponible en http://www.portafolio.co/negocios/especial-marcas-en-colombia-2016-500622+

Samper, Luis F.; Giovanucci, Daniele y Marques Vieira, Luciana. The powerful role of intangibles in coffee value chains: Emerging solutions to grow value and distribute it more equitably [wIPo Economic Research Working Paper n. ${ }^{\circ} 39$ ], noviembre de 2017.

SASB (Sustainability Accounting Standards Board). Food Retailers \& Distributors. San Francisco CA: SASB, 2015. Disponible en http://www.sasb.org/wp-content/ uploads/2015/04/CN0401_Food-Retailers-Distributors_FINAL.pdf. (Accesado el 14 de noviembre de 2016).

Shrum, Larry J. Media consumption and perceptions of social reality. Effects of underlying processes. En J. Bryant \& D. Zillman. Media effects: Advances in theory and research. NJ: LEA Publishers, 2002. ISBN: 9780805838633.

Slotje, Daniel (ed.). Economic damages in intellectual property. NJ: John Wiley and Sons, 2006. ISBN: 978-0-471-79341-0.

Sustainable Brands. 22 Research Studies Proving the ROI of Sustainability. San Francisco, CA: Sustainable Brands, 2016. Disponible en http://e.sustainablebrands. $\mathrm{com} /$ resources-report-22-research-studies-proving-the-roi-of-sustainability. html?_ga $=1.77425757 .974955321 .14677253368$ iesrc $=c t r$

The Economist. Consumer goods. Invasion of the bottle snatchers: Smaller rivals are assaulting the world's biggest brands. The Economist, July 19th, 2016. Issn: 0013-0613. Disponible en http://www.economist.com/news/ business/21701798-smaller-rivals-are-assaulting-worlds-biggest-brandsinvasion-bottle-snatchers

Torres, Leonidas. La importancia de los activos intangibles en la sociedad del conocimiento. Revista La Propiedad Inmaterial. Bogotá: Universidad Externado de Colombia, 2004, (18), pp. 5-34. Issn: 1657-1959.

wbCSD (World Business Council for Sustainable Development) \& UNEP FI (United Nations Environment Programme Finance Initiative). Translating ESG into sustainable business value: Key insights for companies and investors. Report from an International Workshop Weries of the WBCSD and UNEP FI, UNEP FI: Geneva, Switzerland. Conches-Geneva, Switzerland: UNP and WBCSD, 2010. Disponible en http://www.unepfi.org/fileadmin/documents/translatingESG.pdf 
Williams, FreYa. Green giants. How smart companies turn sustainability into billiondollar business. New York: Amacom, 2015. IsBN: 978-0814436134.

Zadek, Simon. The path to corporate responsibility. Harvard Business Review [en línea]. Boston, MA: Harvard Business School Publishing, 2004, 82. IssN: 0017-8012. Disponible en https://hbr.org/2004/12/the-path-to-corporateresponsibility (accesado el 2 enero de 2017). 